\title{
Blind Channel and Data Estimation Using Fuzzy Logic Empowered Cognitive and Social Information-Based Particle Swarm Optimization (PSO)
}

\author{
Muhammad Asadullah ${ }^{1,2}$, Muhammad Adnan Khan ${ }^{1,3,}{ }^{*}$, Sagheer Abbas ${ }^{1}$, Tahir Alyas ${ }^{3}$, Muhammad Asif Saleem ${ }^{3}$, \\ Areej Fatima ${ }^{1,3}$ \\ ${ }^{1}$ School of Computer Sciences, National College of Business Administration and Economics, E 40/ 1 Shahrah-e-Hazrat Imam Hussain, Block E 1 Gulberg III, Lahore, \\ 54000 Lahore, Punjab, Pakistan \\ ${ }^{2}$ Department of Computer Science \& IT, The University of Lahore, 1 - Km Defence Road, 54000 Lahore, Punjab, Pakistan \\ ${ }^{3}$ Department of Computer Science, Lahore Garrison University, Sector C, Phase VI, DHA, 54000 Lahore, Punjab, Pakistan
}

\section{ARTICLE INFO \\ Article History \\ Received 28 Oct 2019 \\ Accepted 13 Feb 2020 \\ Keywords \\ Multiple Input Multiple Output (MIMO) \\ Orthogonal Frequency Division \\ Multiple Access (OFDMA) \\ Multi-Carrier Code Division \\ Multiple Access (MC-CDMA) \\ Multi User Detection (MUD) \\ Channel State Information (CSI)}

\begin{abstract}
Multiple Input Multiple Output (MIMO) is a technology used to improve the channel capacity of the wireless communication systems. Rapid increase in the number of users has led to data rate demand increased in growing modern wireless communication systems. To overcome this issue, MIMO is being used with several multicarrier techniques like Orthogonal Frequency Division Multiple Access (OFDMA), Multi-Carrier Code Division Multiple Access (MC-CDMA), etc. Multi-user detection (MUD) with artificial intelligence plays a vital role to enhance network capacity to meet the demands of future networks with an increased number of users and multimedia services. Computational intelligence techniques are used in a multicarrier system to boost the process of MUD. Some of the computational intelligence algorithms like Swarm and Evolutionary are stuck in local minima and due to this issue, the overall performance of the network decreases. For the convergence of Swarm intelligence-based solutions, cognitive and social information (CSI) play a vital role. In this research article, the Fuzzy Logic empowered Cognitive and Social Information (FLeCSI) algorithm using a fuzzy logic and swarm intelligence algorithm is proposed. By using social and cognitive information FLeCSI updated each swarm position. After the simulation, it is observed that FLeCSI provides fast convergence and minimize MMSE and BER as compared to techniques used previously for MUD like Fuzzy Logic empowered Opposite Mutant Particle Swarm Optimization (FLOMPSO), Opposite Learning Mutant Particle Swarm Optimization (OLMPSO), Total Opposite Mutant Particle Swarm Optimization (TOMPSO), Partial Opposite Mutant Particle Swarm Optimization (POMPSO), etc.
\end{abstract}

(C) 2020 The Authors. Published by Atlantis Press SARL.

This is an open access article distributed under the CC BY-NC 4.0 license (http://creativecommons.org/licenses/by-nc/4.0/).

\section{INTRODUCTION}

Due to rapidly growing wireless communication systems fast technological advancements are needed to meet the data transmission requirements of developing communication systems. Instead of physical wireline channels, wireless channels are used in the wireless communication system. Different types of services including multimedia communication and voice to data are provided by the wireless communication system. Rapidly growing wireless systems needs rapid technological advancements to enhance the capacity of current wireline systems. Undesired effects can be occurred due to affected wireless signals because of physical properties and complex interaction with the environment.

Due to signal scattering, around obstructing and large objects the electromagnetic waves diffraction some problems that occur between transmitter and receiver channel. Because of this problem signal effect with distortion, different attenuation, phase shift, delays and inference of signal for multipath may be Beneficial or

\footnotetext{
Corresponding author: Email: madnankhan@lgu.edu.pk
}

detrimental. When destructive interface occurs the signal power may be diminished slightly.

For coherent or logically related detection of signal at receiving antenna accurate channel state information (CSI) or channel estimation (CE) is needed for most appropriate and enhancement in performance of wireless communication system. If CSI is not available at the receiver, then differential demodulation technique is used by non-coherent methods for detection and demodulation of a signal transmitted. Non-coherent methods as compared with the coherent detection method, due to the non-coherent detection method loss in SNR cost about 3-4 dB loss. This situation directs the research toward a coherent detection method at the receiver for providing Channel Staten Information (CSI) in wireless communication systems.

For detection of inferring signals compressive sensing is used by multi-user detection (MUD) as receiver technology. Sparse property occurs in transmitting signal vector due to a large number of non-zero elements in a situation in which mostly devices are not inactive state and compressive signal (CS) problem occurs while 
decoding of the transmitted signal at a receiver. Long term evolution is more suitable for a wireless communication system that has a high activity a small number of users.

Research directs toward the enhancement of channel capacity without affecting the quality of service for the modern wireless-based networks for communication. For resolving the issues of channel capacity and enhancing the data rates MIMO technique is more effective [1-4]. In the MIMO method, the estimation of signals at receiver and sender antennas is done [5] and increased the data rate and bandwidth of the channel capacity [6-9].

To enhance the corresponding technique some antennas (transmitter) and beneficiary radio are used. Depending on the amount of data conveyed by the MIMO framework increment, the transmitted information is calculated on several transmission paths [5]. While on the receiving point, some receiver antennas receive information to perform different calculations for the restoration of the information to convert the data into the original form at the receiver [10]. For remote communication, MIMO technology is regarded as midpoint and the amount of information without any additional data transfer capacity and transmitting power because of an increase in the scope and amount of information [11,12].

For enhancing the volume growth for several correspondences, the Orthogonal Frequency Division Multiplexing (OFDM) and multicarrier code division multiple access (MC-CDMA) can also be utilized with medium MIMO innovation technique [5-7,13]. One of the optimal detectors is the maximum likelihood (ML) method but is difficult to use for the achievement of exponential complexity. Minimum mean square error (MMSE) detector/locator in Machine to Machine (M2M) and the Maximum a Posteriori (MAP) or marginal likelihood detectors, the sub-optimal MUD detectors for example null steering or the zero forcing detector are used in a less complex situation. The data sent simultaneously by multiple servers to share multi-channel access on the bases of strategies used for demodulation which is the main issue of the MUD. The last two suboptimal techniques are very simple as they use matrix inversion. Evolutionary algorithms like fuzzy adaptive differential evolution (FADE), repeated weighted boosting search (RBS) and differential evolution algorithms (DEAs) are very useful for MUD and CE. Continues Search Space used for CE and discrete search space for MUD. The Multiuser-MIMO (MU MIMO) broadcasting techniques are most widely used for improvement of the spectral efficiency. The quality of pre-coding transmitting to dominate the multiuser inference degraded because of course knowledge of CSI at the transmitter. Hence, there are chances that the interface from co-scheduled user equipment will affect system throughput.

Therefore, the several new techniques like Particle Swarm Optimization (PSO), Total Opposite Mutant Particle Swarm Optimization (TOMPSO) $[5,7,8,13]$, Partial Opposite Mutant Particle Swarm Optimization (POMPSO), Island Differential Equation (IDE), Genetic Algorithm (GA) and Island GA could be used for enhancing the performance and capacity of modern digital communication system [6,7,13-17].

In correspond for both antennas, i.e. sender and receiver, we performed CE for high data rates in this research. Some distortion added to the sending signal during transmission through the strength of the weakens signal and the channel, due to this scenario the receiver may not be able to get actual transmitted information. For avoidance of said situation, fuzzy logic used to enhance the channel and data estimation procedure $[7,8]$. In this article Fuzzy Logic empowered Cognitive and Social Information (FLeCSI)-based new variant of particle swarm intelligence technique used to fulfill/meet the requirements/demands of future generation communication systems.

In this research work, the MIMO system is used because it consists of a different number of users. It is assumed that the channel is cyclo stationary and flat fading.

The core contributions of the article are enumerated as follows:

(a) Formulate an optimization problem for that the main objective is to minimize MMSE, Minimum Mean Channel Error (MMCE) and Bit Error Rate (BER) for optimum utilization of bandwidth.

(b) For the better approximation of the user data and the channel coefficients, Fuzzy Logic empowered the Cognitive Social Information (FLeCSI) algorithm with two variants FLeCSITOLMPSO and FLeCSI-POLMPSO has been proposed.

(c) Proposed FLeCSI method compared with various state of art algorithms like TOMPSO and POMPSO $[13,18]$ and FLOLMPSO [18]. Simulation results have shown that FLeCSI gives far better results as compared to other algorithms.

The sequence of the article is as follows: Literature review, MIMO system model, the proposed FLeCSI-based optimization problem is expressed in Sections 2, 3, and 4. Section 5 represents the simulation results and discussion. Afterward, the research work conclusion and future work in Sections 6 and 7.

\section{LITERATURE REVIEW}

PSO algorithm has been proposed by $[19,20]$ based on the observation of bird's swarm in search of food. According to the PSO algorithm each bird acts as a particle updating his position on the bases of starting velocity, the last position visited personally, i.e. gathered information about neighboring best position from social activities, i.e. social component and cognitive component. PSO provides faster convergence in some optimization problems as compared to the evolutionary algorithm [21], the second thing is the variation of parameters is less. PSO is sensitive to fall into local optimum points when optimizing complex multidimensional functions that is its major drawback. Some qualities of PSO including greater and easy implementation researchers attracted to work with PSO. Several modifications have been done in the PSO algorithm that is applied in different applications of fuzzy logic, neural network [22] and many more.

For batter control over the velocity of swarm particles and to get overall better performance Inertia factor $(\mathrm{w})$ inducted in standard PSO. On the basis of logistic human population growth, a new Incremental Particle size PSO (IPPSO) has been proposed in which the size increases with every iteration. For achieving an overall improvement in the performance of PSO a fast convergence PSO algorithm in which a new parameter named particle mean dimension has been proposed for the balance of diversity of each swarm particle [23]. The variant of the PSO algorithm that ensures convergence of the searching method with better solutions than standard PSO based on the construction factor approach [24]. Hybrid PSO incorporating dynamic chaos improved the efficiency of the 
algorithm [25]. For stable convergence of complex multidimensional problems, PSO particles ability have been explored by [24].

Exploration and exploitation capacity of GA and PSO have been compared [26]. A novel hybrid algorithm based on PSO and GA have been proposed [27]. Incorporated PSO with the technique of tournament selection where current best particle properties transferred into inferior ones. Suganthan et al. [28] proposed an algorithm named Local Best (LBest) model, in which the whole swarm population divided into a small neighborhood and each neighborhood maintain its LBest itself and is very well as in local optima solution it avoids trapping of the swarm but its convergence rate is very low. An adaptive inertia weight PSO algorithm has been proposed by [29].

A new variant of PSO have been introduced for reaching global optima reduce exploitation time and non-linear variation of inertia weight for improvement of search capacity in multidimensional space has been used [30]. By adding dynamic behavioral inertia factor and mutating the particles of PSO adaptively PSO performance has been improved [31]. An improved PSO technique proposed by [32] used for the identification of parameters in chaotic dynamical systems. Brits et al. [33] proposed the Niche PSO technique used for several global optimum solutions for multimodal optimization. An evolutionary algorithm BSA [34] like PSO while looking for global optima chooses the direction of an individual to move from any particles of the last generation randomly. A number of different unimodal optimization problems have been explored and exploited by using the PSO algorithm [35]. To include the effect of inertia term in PSO [36] studied the trajectories of general swarm particles. A novel reformed modified version of PSO like Cognitive and Social information-based Particle Swarm Optimization (CSI-PSO) proposed by [37] in which instead of the velocity concept of a swarm particle each swarm particle updates its position from its own position in search space using its personal and social information.

\section{SYSTEM MODEL}

Figure 1 shows "n" no. of antennas at the receiver end. After receiving signals frequency down is performed. Remove Cyclic Prefix which adds at the transmitter end and then convert it into Serial to Parallel (S/P). After S|P Fast Fourier Transform (FFT) is performed.

There are $\mathrm{A}$ transmit antennas and $\mathrm{O}$ receiving antennas. The channel implemented is a flat fading channel. During the commu-

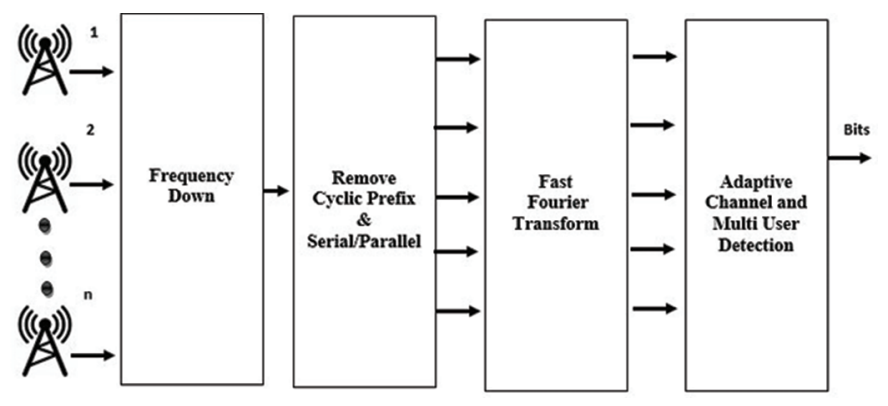

Figure 1 Proposed hybrid computational intelligence approach-based multiple input and multiple output receiver system model. nication process of S symbols, the channel is expected to be stationary. The received signal at receive antenna $o$ is as [1]:

$$
r_{o}(i)=\sum_{a=1}^{A} n_{o . a} e_{a}(i)+v_{o}(i)
$$

where $i$ is the index of symbol, $n_{\text {o.a }}$ is the flat fading channel coefficient that links transfer antenna a to receiver antenna $o, e_{a}(i)$ is $\mathrm{i}^{\text {th }}$ symbol transmitted from antenna a taking value from the symbol set $\{-1,+1\}$ of Binary Phase Shift Key (BPSK) and $v_{o}(i)$ is Additive White Gaussian Noise (AWGN) with $\mathrm{F}\left[\left|v_{o}(i)\right|^{2}\right]=2 \sigma_{V}^{2}$.

The MIMO channel equation below will represent the complete system:

$$
r(i)=\mathrm{Ne}(i)+v(i)
$$

where $\boldsymbol{v}(\boldsymbol{i})$ represents AWGN.

$$
\boldsymbol{v}(i)=\left[v_{1}(i) v_{2}(i) \ldots v_{O}(i)\right]^{T}
$$

The transmitted symbol vector is

$$
\boldsymbol{e}(\boldsymbol{i})=\left[e_{1}(i) e_{2}(i) \ldots e_{A}(i)\right]^{T}
$$

and the received signal vector is

$$
\boldsymbol{r}(\boldsymbol{i})=\left[\begin{array}{llll}
r_{1}(i) r_{2}(i) & \ldots & r_{o}(i)
\end{array}\right]^{T}
$$

The channel gain at receive antenna can always be normalized to unity.

$$
\sum_{a=1}^{A}\left|n_{o, a}\right|^{2}=1
$$

where, $N(o, a)=n_{\text {o. } a}$

Now defined a received data matrix with $\mathrm{O} \times \mathrm{V}$ dimensions and transmitted data matrix with $\mathrm{A}^{\star} \mathrm{V}$ dimensions as [1]:

$$
\boldsymbol{R}=[\boldsymbol{r}(1) \boldsymbol{r}(2) \ldots \boldsymbol{r}(S)]
$$

and

$$
\boldsymbol{E}=[\boldsymbol{e}(1) \boldsymbol{e}(2) \ldots e(S)]
$$

respectively. Then the PDF of the received signal matrix $\mathrm{R}$ conditioned on the MIMO channel matrix $\mathrm{H}$ and the transmitted data matrix F can be written as

$$
P_{r o b}\left(\frac{\boldsymbol{R}}{\boldsymbol{N}, \boldsymbol{E}}\right)=\frac{1}{2 \pi \sigma_{v}^{2} O S} e^{-\frac{1}{2 \sigma_{v}^{2}}} \sum_{i=1}^{S}\|r(n)-N e(i)\|^{2}
$$

The ML estimation of the transmitted symbols $\mathrm{E}$ and the MIMO channel matrix $\mathrm{H}$ can be obtained by maximizing the $P_{r o b}\left(\frac{R}{N, E}\right)$ over $\mathrm{N}$ and $\mathrm{E}$ mutually. Equally, the joint ML estimation can be obtained by minimizing the following cost function:

$$
J_{M L}(\tilde{\boldsymbol{E}}, \tilde{\boldsymbol{N}})=\frac{1}{O \times S} \sum_{i=1}^{S}\|\boldsymbol{r}(\boldsymbol{i})-\tilde{\boldsymbol{N}} \tilde{\boldsymbol{e}}(\boldsymbol{i})\|^{2}
$$

Namely, the joint ML CDE is obtained as

$$
J_{M L}(\tilde{\boldsymbol{E}}, \tilde{\boldsymbol{N}})=\arg \left\{\min _{\tilde{T}, \tilde{\boldsymbol{N}}} J_{M L}(\tilde{\boldsymbol{E}}, \tilde{\boldsymbol{N}})\right\}
$$

Equation (7) demonstrates that the search for the optimal joint ML solution is over the discrete space of the transmitted symbols and the continuous space of the MIMO channel matrix mutually. 


\subsection{Improved Cost Function}

Equation (7) can be written as

$$
J_{M L}(\tilde{\boldsymbol{E}}, \tilde{\boldsymbol{N}})=\frac{1}{O * S}\left[\sum_{i=1}^{S} \boldsymbol{r}^{2}(i)-2 \sum_{i=1}^{S} \boldsymbol{r}(i) \tilde{\boldsymbol{N}} \tilde{\boldsymbol{e}}(i)+\sum_{i=1}^{S}\|\tilde{\boldsymbol{N}} \tilde{\boldsymbol{e}}(i)\|^{2}\right]
$$

The procedure above mentioned is included in any MUD and CE for any multi-user MIMO system. MMSE-based batch processing receiver, cost function and derivation is given below [18].

$$
\begin{aligned}
J_{M L}(\tilde{\boldsymbol{E}}, \tilde{\boldsymbol{N}})= & \frac{1}{O * S}\left[\sum_{i=1}^{S} \boldsymbol{r}^{\mathbf{2}}(i)-2 \sum_{i=1}^{S} \boldsymbol{r}(i) \tilde{\boldsymbol{N}} \tilde{\boldsymbol{e}}(i)\right. \\
& \left.+\sum_{i=1}^{S}\|\tilde{\boldsymbol{N}} \tilde{\boldsymbol{e}}(i)\|^{2}\right]
\end{aligned}
$$

In the above equation, "S" Total symbols are transmitted over "O" receive antennas. Then we let,

$$
C_{M L}(\tilde{\boldsymbol{E}}, \tilde{\boldsymbol{N}})=2 \sum_{i=1}^{S} r(i) \tilde{\boldsymbol{N}} \tilde{\boldsymbol{e}}(n)-\sum_{i=1}^{S}\|\tilde{\boldsymbol{N}} \tilde{\boldsymbol{e}}(i)\|^{2}
$$

Putting the values from Equation (1) to (2)

$$
J_{M L}(\tilde{\boldsymbol{E}}, \tilde{\boldsymbol{N}})=\frac{1}{O \times S}\left[\sum_{i=1}^{S} r^{2}(i)-C_{M L}(\tilde{\boldsymbol{E}}, \tilde{\boldsymbol{N}})\right]
$$

Equation (1) can be written as

$$
J_{M L}(\tilde{E}, \tilde{N})=\left[\min _{\tilde{E}, \mathrm{~N}}\left[\sum_{i=1}^{S} \boldsymbol{r}^{2}(i)-C_{M L}(\tilde{E}, \tilde{N})\right]\right]
$$

It is observed that joined ML CDE can be presented as [18]

$$
J_{M L}(\tilde{E}, \tilde{N})=\max _{\tilde{E}, \tilde{N}} C_{M L}(\tilde{E}, \tilde{N})
$$

\section{PROPOSED FUZZY LOGIC EMPOWERED CSI-BASED OPPOSITE SWARM OPTIMIZATION ALGORITHM}

Opposite Swarm Optimization used with fuzzy logic to enhance the velocity with the help of fuzzy logic controller which takes two input parameter as local and global intelligence and provide the cognition and social information. Algorithm performed following different steps for the preparation and evaluation of data.

Step 1: To start and initialize the populaces of data $\mathbf{P}_{\mathbf{a}}=\left\{\mathbf{P}_{\mathrm{a} 1}, \mathbf{P}_{\mathrm{a} 2}\right.$, . $\left.\mathbf{P}_{\mathrm{ae}}\right\}$ and velocity $\mathbf{V}_{\mathbf{d}}$

Step 2: To calculate cost of population utilization.

Step 3: To calculate the value of Lower and Upper Bound $\left(\operatorname{LP}_{b}\right)$ and $\left(\mathrm{UP}_{\mathrm{b}}\right)$ from $\mathrm{P}_{\mathrm{b}}$ independently.

Step 4: To calculate the opposite populace given as:

- Total Opposite Learning

Opposite Data Population

$$
\begin{aligned}
& \mathbf{Q P} \mathbf{P}_{\mathbf{a}}=\left\{\mathbf{Q P} \mathbf{P}_{\mathrm{a} 1}, \mathbf{Q} \mathbf{P}_{\mathrm{a} 2}, \ldots \ldots \ldots \ldots \ldots \ldots . . . . . . . . . \mathbf{P}_{\mathrm{ae}}\right\} \\
& \mathrm{PP}_{\mathrm{ai}}=\left\{\mathrm{PP}_{\mathrm{ai}, 1}, \mathrm{QP}_{\mathrm{ai}, 2}, \ldots \ldots \ldots \ldots \ldots . . . \mathrm{PP}_{\mathrm{ai}, \mathrm{M}}\right\} \\
& \mathrm{PP}_{\mathrm{a}, \mathrm{j}}=u \mathrm{~b}_{\mathrm{p}}+\mathrm{h} \mathrm{b}_{\mathrm{a}}-\mathrm{P}_{\mathrm{a}, \mathrm{j}} \text {. }
\end{aligned}
$$

- Partial Opposite Learning Opposite Data Population

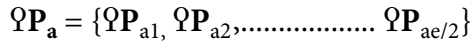

$$
\begin{aligned}
& \mathrm{PP}_{\mathrm{ai}}=\left\{\mathrm{PP}_{\mathrm{ai}, 1}, \mathrm{QP}_{\mathrm{ai}, 2}, \ldots \ldots \ldots \ldots \ldots . . . . . . . \mathrm{PP}_{\mathrm{ai}, \mathrm{M}}\right\} \\
& \mathrm{PP}_{\mathrm{ai}, \mathrm{j}}=\mathrm{ub} \mathrm{b}_{\mathrm{p}}+h \mathrm{~b}_{\mathrm{a}}-\mathrm{P}_{\mathrm{ai}, \mathrm{j}} \text {. }
\end{aligned}
$$

Step 5: To calculate the appropriateness of Opposite Populations $(\mathrm{PP})$

Step 6: Choose the value of data population in Local best particle $\mathrm{M}_{\mathrm{bpa}}$ from $\mathbf{P}_{\mathbf{a}}$ and $\mathbf{Q} \mathbf{P}_{\mathbf{a}}$ and Global best particle of $\mathbf{G}_{\mathrm{bpa}}$ from $\mathbf{P}_{\mathbf{a}}$ and $\mathbf{Q P} \mathbf{P}_{\mathbf{a}}$.

After performing the above-mentioned steps, select and update the velocity of Global best particle for data population

Step 7: Calculate Local \& Global Intelligence:

$$
(\mathrm{LI})=\mathrm{P}_{\mathrm{ij}}-\mathrm{LP}_{\mathrm{bij}(\mathrm{n}-1)} \text { and }(\mathrm{GI})=\mathrm{P}_{\mathrm{ij}}-\mathrm{GP}_{\mathrm{bij}(\mathrm{n}-1)}
$$

$[\mathrm{FLCO}, \mathrm{FLSO}]=$ FLC $(\mathrm{LI}, \mathrm{GI})$

$\mathrm{V}_{\mathrm{dij}(\mathrm{n})}=\mathrm{V}_{\mathrm{dij}(\mathrm{n}-1)}$ FLCO (Local Intelligence) LI + FLSO (Global intelligence)

Step 8: Calculate the data population particle of fitness and update the value of $\mathrm{P}_{\mathrm{a}}$

Step 9: The same iterations perform for the next received signal until achieved the desired MSE or NoC.

Step 10: Finally Stop

The membership function of input and output variables used in the proposed system mathematically and graphically are presented below (Table 1).

\subsection{Fuzzy Propositions}

A fuzzy proposition represents the union, intersections and complement by using the connectives "or," "and" \& "not." Finally, the fuzzy propositions can be written as

$$
t: l i \times g i \rightarrow[F L C O, F L S O]
$$

Here, $l i$, gi, FLCO and FLSO represent Local Intelligence, Global Intelligence, Fuzzy Logic empowered Cognition and Fuzzy Logic empowered Social information respectively. All values are represented in the range of 0 to 1 in both the I/O variable. Equation (14) shows the t-norm function for the final layer as

$$
t:[0,1] \times[0,1] \rightarrow[[0,1],[0,1]]
$$

Proposed fuzzy inference system along with membership function defined as

$$
t\left[\mu_{L}(l i), \mu_{G}(g i)\right]=\min \left[\mu_{L}(l i), \mu_{G}(g i)\right]
$$

Equation (15) can be written as

$$
\mu_{L \cap G}(l i, g i)=t\left[\mu_{L}(l i), \mu_{G}(g i)\right]
$$

From Equations (15) and (16)

$$
\mu_{L \cap G}(l i, g i)=\left[\mu_{L}(l i), \mu_{G}(g i)\right]
$$


Table 1 Membership function variables of input/output used in the proposed Fuzzy Logic empowered Cognitive and Social Information (FLeCSI)-OSO-based system.

Sr \#

\section{Input/Output}

Variables
Representation of Membership

Functions (MF) Mathematically
Representation of Membership

Functions (MF) Graphically

$\mu L i, \operatorname{small}(l)$

$$
=\left(\left(1,1-2\left(\frac{l^{2}+9}{9}\right), 2\left(\frac{l^{2}}{9}\right)\right), 0\right)
$$

$\mu L i, \operatorname{Medium}(l)$

$$
\begin{aligned}
\text { LocalInt }=\operatorname{LI} \mu_{L i}(l) \quad= & \left(2\left(\frac{l^{2}+9}{9}\right), 1-2\left(\frac{l^{2}}{9}\right), 1,1\right. \\
& \left.-2\left(\frac{l^{2}+4}{9}\right), 2\left(\frac{l^{2}+25}{9}\right), 0\right)
\end{aligned}
$$

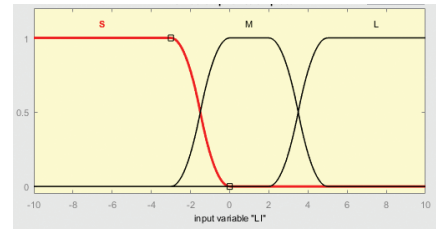

$\mu$ Li, Large (l)

$$
\left.=\left(2\left(\frac{l^{2}+4}{4}\right), 1-2\left(\frac{l^{2}-25}{9}\right), 1\right), 0\right)
$$

$\mu G i, \operatorname{small}(g)$

$$
=\left(\left(1,1-2\left(\frac{g^{2}+9}{9}\right), 2\left(\frac{g^{2}}{9}\right)\right), 0\right)
$$

$\mu G i, \operatorname{Medium}(g)$

$2 \quad$ GlobalInt $=\mathrm{GI} \mu_{G i}(g)$

$$
\begin{aligned}
= & \left(2\left(\frac{g^{2}+9}{9}\right), 1-2\left(\frac{g^{2}}{9}\right), 1,1\right. \\
& \left.-2\left(\frac{g^{2}+4}{9}\right), 2\left(\frac{g^{2}+25}{9}\right), 0\right)
\end{aligned}
$$

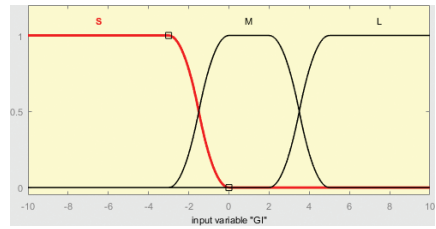

$\mu$ Gi, Large $(g)$

$$
\left.=\left(2\left(\frac{g^{2}+4}{4}\right), 1-2\left(\frac{g^{2}-25}{9}\right), 1\right), 0\right)
$$

$\mu F c, \operatorname{low}(F c)$

$$
=\left(\left(0,\left(\frac{0.16-f_{c}}{0.16-0.07}\right)\right), 0\right)
$$

$\mu F c$, medium $(F c)$

$3 \quad \mathrm{FLCO}=\mathrm{FC} \mu_{\mathrm{Fc}}(\mathrm{fc})$

$$
=\left(\left(0,\left(\frac{f c+0.07}{0.16-0.07}\right),\left(\frac{0.062-f_{c}}{0.62-0.53}\right)\right), 0\right)
$$

$\mu F c, \operatorname{high}(F c)$

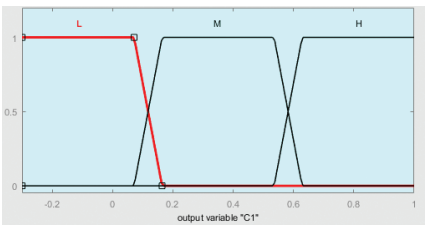

$$
=\left(\left(0,\left(\frac{f c-0.53}{0.62-0.53}\right)\right), 1\right)
$$

$\mu F s, \operatorname{low}(F s)$

$$
=\left(\left(0,\left(\frac{0.16-f_{s}}{0.16-0.07}\right)\right), 0\right)
$$

$\mu F s, \operatorname{medium}(F s)$

4

$\mathrm{FLSO}=\mathrm{FS} \mu_{\mathrm{Fs}}(\mathrm{fs})$

$$
=\left(\left(0,\left(\frac{f s+0.07}{0.16-0.07}\right),\left(\frac{0.062-f s}{0.62-0.53}\right)\right), 0\right)
$$

$\mu F s, \operatorname{high}(F s)$

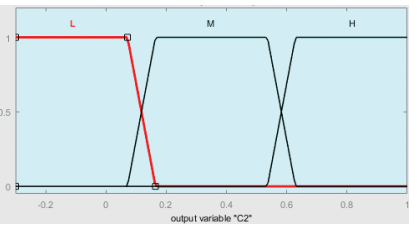

$$
=\left(\left(0,\left(\frac{f_{s}-0.53}{0.62-0.53}\right)\right), 1\right)
$$




\subsection{Lookup Table}

Table 2 shows the lookup table for a proposed system that contains 9 I/O fuzzy rules.

Nine rules of Fuzzy at the final layer denoted by $\operatorname{Rcp}^{\mu}$, while $(1 \leq \mu$ $\leq 9)$.

$\mathrm{Rcp}^{4}=$ For Medium local intelligence \& Small global intelligence THE RESULT IS medium cognitive information and high social information.

$\mathrm{Rcp}^{5}=$ For Medium value of both $\mu_{L}(l i) \& \mu_{G}(g i)$ THE RESULT IS also medium value of both (cognitive and social) information.

$\mathrm{Rcp}^{6}=$ For Medium $\mu_{L}(l i)$ \& Large $\mu_{G}(g i)$ THE RESULT IS medium cognitive information and low social information.

$\mathrm{Rcp}^{9}=$ For Large value of both $\mu_{L}(l i) \& \mu_{G}(g i)$ THE RESULT IS low cognitive information and low social information.

At the final layer nine Fuzzy rules denoted by $\operatorname{Rcp}^{\mu}$, while $(1 \leq$ $\mu \leq 9)$.

\subsection{Inference Engine}

The core part of Fuzzy inference is the fuzzy logic operators, membership functions and IF-THEN rules. Union operator is used for combining the rules of fuzzy relations.

Proposed FLeCSI-based expert system IF-THEN rules be denoted as $R \mathrm{pq}^{\mu}$ of the final layer; which is,

$$
R \mathrm{pq}^{\mu}=L^{\mu} \times G^{\mu} \rightarrow U_{v c 1}^{\mu}, U_{v c 2}^{\mu}
$$

Equation (18) can be written as

$$
\mu_{L \cap G}(l i, g i)=\mu_{L}(l i) \cap \mu_{G}(g i)
$$

The single fuzzy relation using the rules of the final layer is inferred as

$$
R_{9}=\cup_{\mu=1}^{9} R q^{\mu}
$$

Table 2 Lookup table for proposed FLeCSI-OSO-based MC-CDMA system.

\begin{tabular}{l|c|c|c|c}
\hline Rules & $\begin{array}{c}\text { Local } \\
\text { Intelligence }\end{array}$ & $\begin{array}{c}\text { Global } \\
\text { Intelligence }\end{array}$ & $\begin{array}{c}\text { Cognitive } \\
\text { Information }\end{array}$ & $\begin{array}{c}\text { Social } \\
\text { Information }\end{array}$ \\
\hline 1 & Small & Small & High & High \\
2 & Small & Medium & High & Medium \\
3 & Small & Large & High & Low \\
4 & Medium & Small & Medium & High \\
5 & Medium & Medium & Medium & Medium \\
6 & Medium & Large & Medium & Low \\
7 & Large & Small & Low & High \\
8 & Large & Medium & Low & Medium \\
9 & Large & Large & Low & Low \\
\hline
\end{tabular}

FLeCSI, Fuzzy Logic empowered Cognitive and Social Information; MC-CDMA, MultiCarrier Code Division Multiple Access.
Consider $i^{\text {th }}$ and $u$ be fuzzy set and fuzzy inference engine of input and output individually. We get the output fuzzy inference engine to view IF-THEN rule $\mathrm{R}_{9}$ by using the generalized modus ponens as

$$
\begin{aligned}
& \mu_{\text {SlownMediumnHigh }}\left(\mathrm{u}_{c_{1}}, \mathrm{u}_{c_{2}}\right) \\
& \quad=\sup _{i \in(L, G)} t\left[\mu_{i}(l i, g i),\left\{\mu_{R_{9}}\left(l_{i}, g_{i}, U_{v c 1}\right) \mu_{R_{9}}\left(l_{i}, g_{i}, U_{v c 2}\right)\right\}\right]
\end{aligned}
$$

The equation of Product Inference Engine (PIE) can be written as

$$
\begin{aligned}
& \mu_{\xi 1} \text { FLCO, } \mu_{\xi_{2}} \text { FLSO } \\
& \quad=\left[\sup _{i \in(L, G)}\left(\prod_{j=1}^{9}\left(\mu_{L, G}\left(l_{j}, g_{j}\right), \mu_{\beta_{1 i} \beta_{2 i}, \beta_{3 i}}\left(b_{1}, b_{2}, b_{3}\right)\right)\right)\right]
\end{aligned}
$$

\subsection{De-Fuzzifier}

De-fuzzier of the proposed system specifies the $\Psi^{*}$ by the membership function of $\Psi$, i.e.

$$
\begin{aligned}
& {\left[\psi_{\xi 1}{ }^{*}, \psi_{\xi 2}{ }^{*}\right]} \\
& \quad=\left[\frac{\int_{a}^{b} \xi 1 \mu_{\xi 1}(\xi 1) d \xi 1}{\int_{a}^{b} \mu_{\xi 1}(\xi 1) d \xi 1}, \frac{\int_{a}^{b} \xi 2 \mu_{\xi 2}(\xi 2) d \xi 2}{\int_{a}^{b} \mu_{\xi 2}(\xi 2) d \xi 2}\right]
\end{aligned}
$$

The de-fuzzifier of the proposed FLeCSI-based system is shown below in Figures 2 and 3.

Figure 2 shown that if $\mu_{L}(l i)$ is small, medium or large for any value of $\mu_{G}(g i)$ then cognitive information is also low, medium or high, respectively.

Similarly, it also observed from Figure 3 if $\mu_{G}(g i)$ is small, medium or large for any value of $\mu_{L}$ (li) then social information is also low, medium or high, respectively.

\subsection{Lookup Diagrams}

Figures 4-7 shows the lookup diagrams for Proposed Fuzzy Logic empowered CSI-based Swarm Optimization with all possible cases

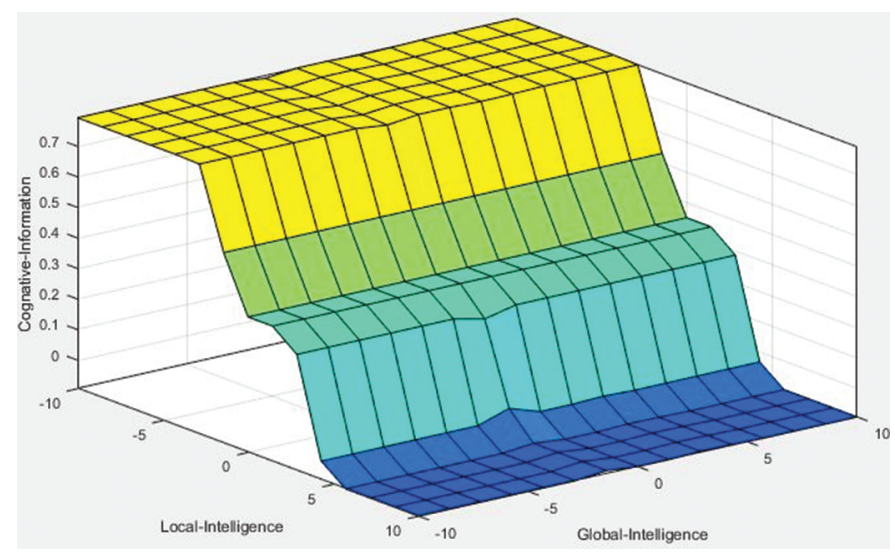

Figure 2 Proposed Fuzzy Logic empowered Cognitive and Social Information (FLeCSI)-based rule surface for cognitive information of local intelligence and the global intelligence. 
of the Updated Cognitive and Social Information. Figure 4 shows that if $\mu_{L}(l i)$ is small \& $\mu_{G}(g i)$ is large the cognitive information is high and social information is low.

Figure 5 shows that if $\mu_{L}(l i)$ is large and $\mu_{G}(g i)$ is small the cognitive information is low and social information is high.

Figure 6 shows that if both $\mu_{L}(l i)$ and $\mu_{G}(g i)$ are medium then both CSI are also medium.

Figure 7 shows that if both $\mu_{L}(l i)$ \&and $\mu_{G}(g i)$ are small then CSI are also high.

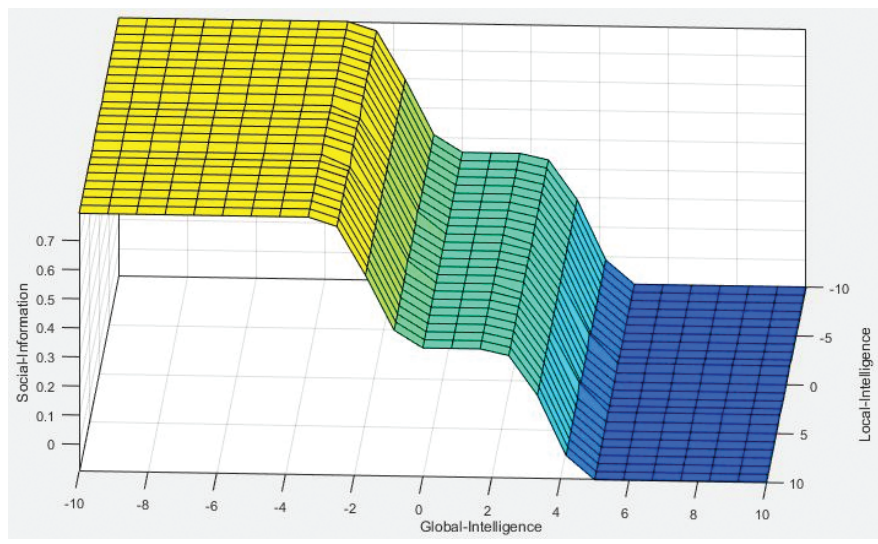

Figure 3 Proposed Fuzzy Logic empowered Cognitive and Social Information (FLeCSI)-based rule surface for social information of local intelligence and the global intelligence.
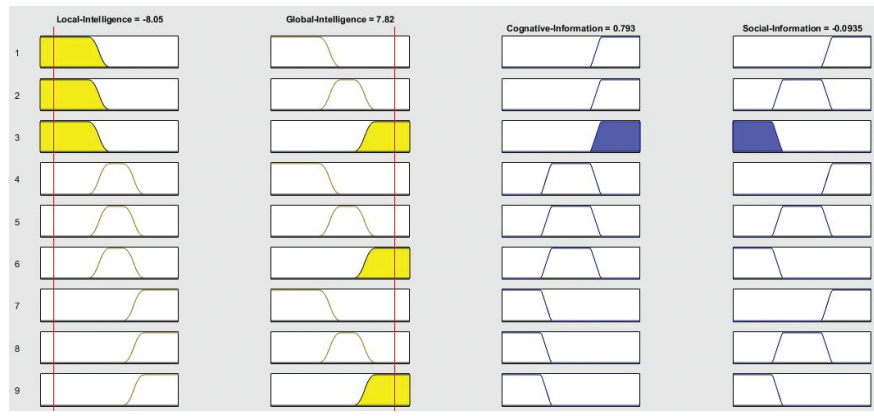

Figure 4 Proposed Fuzzy Logic empowered Cognitive and Social Information (FLeCSI)-based lookup diagram for high CI and low SI.
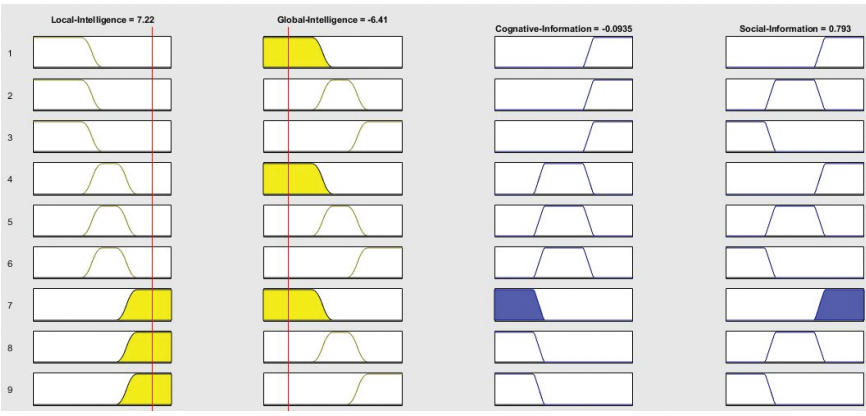

Figure 5 Proposed Fuzzy Logic empowered Cognitive and Social Information (FLeCSI)-based lookup diagram for low CI and high SI.

\subsection{Results and Discussions}

For the implementation of the Blind Channel and Data Estimation (BCDE) MIMO system, BPSK signaling scheme is used at data sequence length $\mathrm{Q}=50$. All transmitters are equipped with $\mathrm{A}=3$ to transmit antennas and $\mathrm{O}=3$ antenna used for the base stations. Implementation of the Rayleigh flat fading channel with four paths. The transmitter carrier frequency 900 Megahertz, which corresponds to the Doppler frequency of 25 Hertz also moving at the speed of $30 \mathrm{~km} /$ per hour. For simulation $3^{\star} \mathrm{k}$ MIMO channel is used having $\mathrm{k}=10$ users with a data population of 100 and 5 no. of cycles for both algorithms. Channel population size is $5^{\star} P_{h}$ where $P_{h}$ represents the size of the channel matrix.

However, the simulation performance is measured by the MMCE, which is:

$$
M M C E=\frac{1}{A * O} \sum_{a=1}^{A} \sum_{o=1}^{O}\left|n_{a, o}-\tilde{N}^{*}(a, o)\right|
$$

The performance and comparison of Proposed FLeCSI based on both variants with previous algorithms are represented in Tables 3 and 4 for channel and data estimation of MIMO system with respect to Number of Cycles (NoCs) v/s MMSE, Miss Rate/BER and MMCE, respectively.

Table 3 shown that Conventional POMPSO and Conventional TOMPSO proposed in $[13,18]$ gives $10^{-3}$ and $10^{-5.4}$ MMSE at 160th and 180th NoCs, respectively. FL-POLMPSO and FL-TOLMPSO proposed in [18] converges $10^{-3.5}$ and $10^{-5.5}$ MMSE at 150th and 160th NoCs, respectively. And Proposed FLeCSI-POLMPSO and FLeCSI- TOLMPSO gives $4.6017^{\star} 10^{-6}, 4.6016^{\star} 10^{-6}$ MMSE
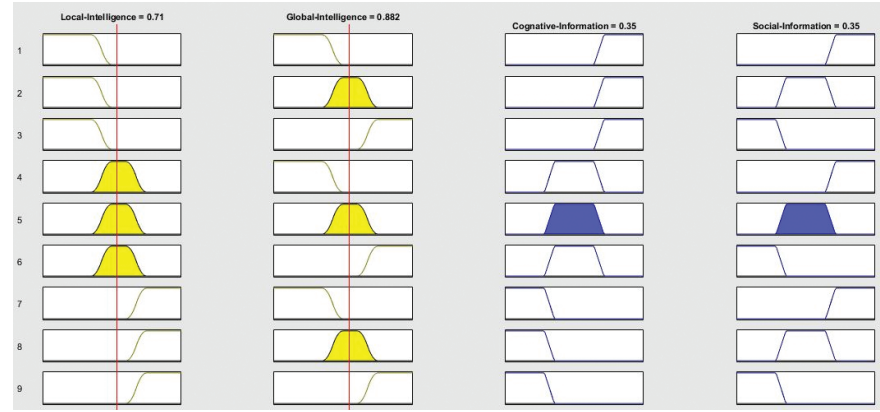

Figure 6 Proposed FLeCSI-based lookup diagram for medium CI and medium SI.

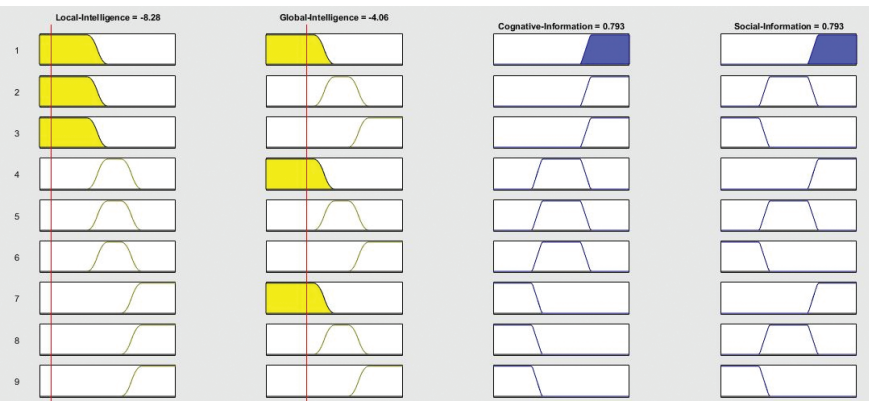

Figure 7 Proposed Fuzzy Logic empowered Cognitive and Social Information (FLeCSI)-based lookup diagram for high CI and high SI. 
Table 3 Comparison with the state-of-the-art method w.r.t NoC vs MMSE.

\begin{tabular}{lcc}
\hline Method & $\begin{array}{c}\text { Number of } \\
\text { Cycles }\end{array}$ & $\begin{array}{c}\text { Min Mean Square } \\
\text { Error (SNR = 25dB) }\end{array}$ \\
\hline POMPSO [13,18] & 160 & $10^{-3}$ \\
TOMPSO [13,18] & 180 & $10^{-5.4}$ \\
FL-POLMPSO [18] & 150 & $10^{-3.5}$ \\
FL-TOLMPSO [18] & 160 & $10^{-5.5}$ \\
Proposed FLeCSI-POLMPSO & 117 & $4.6017 \times 10^{-6}$ \\
Proposed FLeCSI- TOLMPSO & 110 & $4.6016 \times 10^{-6}$ \\
\hline
\end{tabular}

NoC, Number of Cycles; MMSE, Minimum Mean Square Error; POMPSO, Partial Opposite Mutant Particle Swarm Optimization; TOMPSO, Total Opposite Mutant Particle Swarm Optimization; FLeCSI, Fuzzy Logic empowered Cognitive and Social Information.

Table 4 Comparison with the state-of-the-art method w.r.t NoC vs MMCE \&and miss rate.

\begin{tabular}{lcc}
\hline Method & $\begin{array}{c}\text { Minimum Mean } \\
\text { Channel Error }(\text { SNR } \\
\text { = 25dB, NoC }=\mathbf{2 0 0})\end{array}$ & $\begin{array}{c}\text { Miss Rate/Bit Error } \\
\text { Rate }(\text { SNR = 25dB, } \\
\text { NoC = 180) }\end{array}$ \\
\hline POMPSO [13,18] & $10^{-2}$ & $1 \times 10^{-3}$ \\
TOMPSO [13,18] & $10^{-4}$ & $3.205 \times 10^{-4}$ \\
FL-POLMPSO [18] & $10^{-3}$ & $3.8656 \times 10^{-6}$ \\
FL-TOLMPSO [18] & $10^{-4.2}$ & $3.3633 \times 10^{-6}$ \\
Proposed FLeCSI- & $3.1944 \times 10^{-5.1}$ & $1.523 \times 10^{-6.5}$ \\
POLMPSO & & \\
Proposed FLeCSI- & $3.001 \times 10^{-5.1}$ & $1.5017 \times 10^{-6.6}$ \\
TOLMPSO & & \\
\hline
\end{tabular}

NoC, Number of Cycles; MMSE, Minimum Mean Square Error; POMPSO, Partial Opposite Mutant Particle Swarm Optimization; TOMPSO, Total Opposite Mutant Particle Swarm Optimization; FLeCSI, Fuzzy Logic empowered Cognitive and Social Information.

at 117 th, 110th NoCs, respectively. It clearly observed Proposed FLeCSI based on both variations give attractive results as compared to previously published approaches in $[13,18]$.

Table 4 shows the performance of proposed FLeCSI based on both variants in terms of MMCE and Mis Rate. The SNR is fixed to $25 \mathrm{~dB}$ for both parameters (MMCE and Mis Rate). The NoCs is also fixed to 200 for MMCE and 180 for Miss Rate. It observed that Conventional POMPSO and Conventional TOMPSO proposed in $[13,18]$ gives $10^{-2}, 10^{-4}$ MMCE and $10^{-3}, 3.205^{\star} 10^{-4}$ Miss Rate, respectively. FL-POLMPSO and FLTOLMPSO proposed in [35] converges $10^{-3}, 10^{-4.2}$ MMCE and $3.8656^{\star} 10^{-6}, 3.3633^{\star} 10^{-6}$ Miss Rate, respectively. And Proposed FLeCSI-POLMPSO and FLeCSI-TOLMPSO gives $3.1944^{\star} 10^{-5.1}$, $3.001^{\star} 10^{-5.1}$ MMCE and $1.523^{\star} 10^{-6.5}, 1.5017^{\star} 10^{-6.6}$ Miss Rate, respectively. It clearly observed Proposed FLeCSI based both variations give attractive results as compared to previously published approaches in $[13,18]$ in terms of MMCE and Miss Rate.

\section{CONCLUSION}

Multiple Input Multiple Output (MIMO) technology is used for the wireless communication systems, when it comes to the capacity development of the channel. As the dynamic environment and growing number of users, data rate demand increased in growing modern wireless communication systems. MUD with artificial intelligence plays a vital role to enhance network capacity to meet the demands of future networks with an increased number of users and multimedia services. The proposed FLeCSI-based MIMO receiver system is fast enough to fulfill the needs of a future generation growing networks. FLeCSI provides more attractive results as compared to other suboptimal-based previous techniques It is also observed that the proposed FLeCSI-based receiver system minimizes BER and MMSE as compared to other techniques like FL-OMPSO, FL-POLMPSO, FL-TOLMPSO, TOMPSO, etc. discussed previously.

\section{FUTURE WORK}

In this article, the Selective fading channel is ignored. Typ2 fuzzy may be used for updating the Cognition and Social information of swarm intelligence. Deep machine learning may be explored on CE along MUD for better results. The application of this proposed FLeCSI can also be applied to medical imaging, channel equalization, spectrum sensing in cognitive radio, etc. The complexity comparison of proposed FLeCSI and their solution can be another point of research.

\section{DATA AVAILABILITY}

The simulation files to support the findings of this study are available from the corresponding author upon request.

\section{CONFLICT OF INTEREST}

The authors declare that there are no conflicts of interest regarding the publication of this article.

\section{AUTHORS' CONTRIBUTIONS}

Muhammad Asadullah, Muhammad Adnan Khan \& Sagheer Abbas proposed the Improved Mathematical System Model \& Algorithm. Muhammad Adnan khan, Muhammad Asadullah \& Tahir Alyas performed the MATLAB Simulations. Muhammad Asadullah, Muhammad Asif Saleem and Areej Fatima contributed in writing-original draft preparation. Muhammad Adnan Khan and Sagheer Abbas performed revision and improve the quality of the draft. All authors have read and agreed to the published version of the manuscript.

\section{Funding Statement}

This research received no external funding.

\section{ACKNOWLEDGMENTS}

The authors would like to express their deepest gratitude to Dr. Shazia Saqib from Lahore Garrison University for the helpful suggestions during proof read this article.

\section{REFERENCES}

[1] T. Abdelkader, K. Mokhtar, O. Abdelaziz, et al., New space-time coding for joint blind channel estimation and data detection through time-varying MIMO channels, Int. J. Comput. Sci. Issues. 11 (2014), 33-38. http://ijcsi.org/papers/IJCSI-11-5-2-33-40.pdf

[2] M. Abuthinien, S. Chen, A. Wolfgang, L. Hanzo, et al., Joint maximum likelihood channel estimation and data detection for MIMO systems, in IEEE International Conference on Communications ICC’07, Glasgow, UK, 2007, pp. 5354-5358. 
[3] A. Mezghani, A.L.S. Hurst, Blind estimation of sparse multi-user massive MIMO channels, in 21st International ITG Workshop on Smart Antennas, Proceedings of VDE, Berlin, Germany, 2017, vol. 2, pp. 2-6. http://arxiv.org/abs/1612.00131

[4] M.N. Seyman, N. Taspinar, Symbol detection using the differential evolution algorithm in MIMO-OFDM systems, Turk. J. Electr. Eng. Comput. Sci. 21 (2013), 373-380.

[5] M. Zubair, M.A. Choudhry, A. Naveed, I.M. Qureshi, et al., Joint channel and data estimation using particle swarm optimization, IEICE Trans. Commun. 91 (2008), 3033-3036.

[6] M.A. Khan, M. Umair, M.A.S. Choudhry, et al., GA based adaptive receiver for MC-CDMA system, Turk. J. Electr. Eng. Comput. Sci. 23 (2015), 2267-2277.

[7] M.A. Khan, M. Umair, M.A.S. Choudhry, et al., Island differential evolution based adaptive receiver for MC-CDMA system, in IEEE International Conference on Information and Communication Technologies (ICICT), Karachi, Pakistan, 2015, pp. 1-6.

[8] M.A. Khan, A. Nasir, M. Umair, S. Abbas, et al., Time complexity of TOMPSO algorithm, in 16th International Conference on Statistical Sciences: Advances in Statistics and Data Management: It's Role in National Growth and Socio-Economic Developments, 2018, vol. 32, pp. 89-100.

[9] P.W. Raut, S.L. Badjate, MIMO-future wireless communication, Int. J. Innov. Technol. Explor. Eng. 2 (2013), 102-106.

[10] M. Biguesh, A.B. Gershman, Training-based MIMO channel estimation: a study of estimator tradeoffs and optimal training signals, IEEE Trans. Signal Process. 54 (2006), 884-893.

[11] P. Chen, H. Kobayashi, Maximum likelihood channel estimation and signal detection for OFDM systems, in IEEE International Conference on Communication, 2002, vol. 3, pp. 1640-1645.

[12] C. Pirak, Z.J. Wang, K.R. Liu, S. Jitapunkul, et al., Optimum power allocation for maximum-likelihood channel estimation in spacetime coded MIMO systems, in 2006 IEEE International Conference on Acoustics, Speech and Signal Processing, ICASSP 2006 Proceedings, Toulouse, France, 2006, vol. 4, pp. 4-6.

[13] M.A. Khan, Multiuser Detection Using Computational Intelligence in Multi-Carrier Communication Systems, ISRA University, Islamabad Campus, Pakistan, 2016. http://prr.hec.gov.pk/ jspui/handle/123456789//8007

[14] M. Umair, M.A. Khan, M.A.S. Choudhry, et al., Island genetic algorithm based MUD for MC-CDMA system, in 6th IEEE International Conference on Information and Communication Technologies, Karachi, Pakistan, 2015, pp. 7-12.

[15] N. Ali, M.A. Khan, M. Adeel, M. Amir, et al., Genetic algorithm based adaptive receiver for MC-CDMA system with variation in mutation operator, Int. J. Comput. Sci. Inf. Secur. USA. 14 (2016), 215-222. https://sites.google.com/site/ijcsis/vol14-no-9-sep-2016

[16] M. Umair, M.A. Khan, M.A.S. Choudhry, et al., Piranha fish optimization for multi-user detection in OFDMA system, Int. J. Adv. Appl. Sci. 3 (2016), 35-40.

[17] M. Umair, M.A. Khan, M.A.S. Choudhry, et al., GA backing to STBC based MC-CDMA systems, in 4th IEEE International Conference on Intelligent Systems, Modelling, and Simulation, Bangkok, Thailand, 2013, pp. 503-506.

[18] M. AsadUllah, M.A. Khan, S. Abbas, A. Athar, S.S. Raza, G. Ahmad, et al., Blind channel and data estimation using fuzzy logic-empowered opposite learning-based mutant particle swarm optimization, Comput. Intell. Neurosci. 2018 (2018), 1-12.
[19] R.C. Eberhart, J. Kennedy, A new optimizer using particle swarm theory, in Proceedings of the Sixth International Symposium on Micro Machine and Human Science, Nagoya, Japan, 1995, pp. 39-43.

[20] J. Kennedy, R.C. Eberhart, Particle swarm optimization, in Proceedings of the IEEE International Conference on Neural Networks, Piscataway, NJ, USA, 1995, vol. 4, pp. 1942-1948.

[21] J. Kennedy, R.C. Eberhart, Swarm Intelligence, Morgan Kaufmann Publishers, Massachusetts, USA, 2001.

[22] H.Z. Wei, C. Yang, L. Gao, X. Yao, R.C. Eberhart, Y. Shi, et al., Extracting rules from fuzzy neural network by particle swarm optimization, in Proceedings of IEEE International Conference on Evolutionary Computation, Anchorage, AK, USA, 1998, pp. 74-77.

[23] A. Sahu, S.K. Panigrahi, S. Pattnaik, et al., Fast convergence particle swarm optimization for functions optimization, Procedia Technol. 4 (2012), 319-324.

[24] M. Clerc, J. Kennedy, The particle swarm explosion, stability and convergence in a multidimensional complex space, IEEE Trans. Evol. Comput. 6 (2002), 58-73.

[25] B. Liu, L. Wang, Y.H. Jin, F. Tang, D.X. Huang, et al., Improved particle swarm optimization combined with chaos, Chaos Soliton. Fract. 25 (2005), 1261-71.

[26] R.C. Eberhart, Y. Shi, Comparison between genetic algorithms and particle swarm optimization, in Evolutionary Programming VII: Proceedings of the Seventh Annual Conference on Evolutionary Programming, San Diego, CA, USA, 1998, pp. 611-616.

[27] X.H. Shi, Y.C. Liang, H.P. Lee, C. Lu, L.M. Wang, et al., An improved GA and a novel PSO-GA-based hybrid algorithm, Inf. Process. Lett. 93 (2005), 255-261.

[28] P.N. Suganthan, Particle swarm optimizer with neighborhood operator, in Proceedings of the 1999 Congress on Evolutionary Computation, CEC'99, Washington, DC, USA, 1999, pp. 1958-1961.

[29] A. Nickabadi, M.M. Ebadzadeh, R. Safabakhsh, et al., A novel particle swarm optimization algorithm with adaptive inertia weight, Appl. Soft Comput. 11 (2011), 3658-3670.

[30] A. Chatterjee, P. Siarry, Nonlinear inertia weight variation for dynamic adaptation in particle swarm optimization, Comput. Oper. Res. 33 (2006), 859-871.

[31] A. Alfi, PSO with adaptive mutation and inertia weight and its application in parameter estimation of dynamic systems, Acta Automat. Sin. 37 (2011), 541-549.

[32] H. Modares, A. Alfi, M.M. Fateh, et al., Parameter identification of chaotic dynamic systems through an improved particle swarm optimization, Expert Syst. Appl. 37 (2010), 3714-3720.

[33] P. Civicioglu, Backtracking search optimization algorithm for numerical optimization problems, Appl. Math. Comput. 219 (2013), 8121-8144.

[34] Y. Shi, R.C. Eberhart, An empirical study of particle swarm optimization, in Proceedings of the IEEE Congress on Evolutionary Computation, Piscataway, NJ, USA, 1999, pp. 1945-1960.

[35] F.V. Bergh, A.P. Engelbrech, A study of particle swarm optimization particle trajectories, Inf. Sci. 176 (2006), 937-971.

[36] D. Tripathi, U. Jena, Cognitive and social information based PSO, Int. J. Eng. Sci. Technol. 8 (2016), 64-67.

[37] R. Brits, A. Engelbrecht, F.V. Bergh, et al., Locating multiple optima using particle swarm optimization, Appl. Math. Comput. 189 (2007), 1859-1883. 